\title{
Higher-order Bessel like beams with z-dependent cone angles
}

\author{
Y.Ismail $^{\mathrm{a}, \mathrm{b}}$, G Barnes $^{\mathrm{b}}$, T Mhlanga $^{\mathrm{b}}, \mathrm{V}$. Belyi ${ }^{\mathrm{d}}$, A. Forbes $^{\mathrm{a}, \mathrm{b} \dagger}$ \\ ${ }^{a}$ CSIR National Laser Centre, Box 395, Pretoria 0001, South Africa \\ ${ }^{\mathrm{b}}$ School of Physics, University of KwaZulu-Natal, Private Bag X54001, Durban 4000, South Africa \\ ${ }^{\mathrm{d}}$ B.I. Stepanov Institute of Physics, NASB, 68 Nezavisimosti ave., 220072 Minsk, Belarus
}

\begin{abstract}
As in the case of zero-order Bessel beam being produced by illuminating an axicon with a Gaussian beam, higher-order Bessel beams are generated by substituting the Gaussian beam with a Laguerre Gaussian (LG) beam. These beams hold similar properties to zero-order Bessel beams except they carry orbital angular momentum (OAM). They also undergo an abrupt spatial transformation at the boundary of their non-diffracting regime whereby the near-field intensity distribution is a Bessel function which transforms into an annular ring (far-field profile). This can be considered a disadvantage to such beams in comparison to a Gaussian beam that is propagation invariant. By using a double axicon lens system this Bessel beams with z-dependent cone angles can be produced however at the expense of its non-diffracting nature. Here we outline an optical design to produce higher-order Bessel-like beams with z-dependent cone angles that will retains its spatial distribution as $\mathrm{z} \rightarrow \infty$.
\end{abstract}

Keywords: Higher-order Bessel beam, Laguerre Gaussian beams, axicon

\section{INTRODUCTION}

Since the inception of zero-order Bessel beams being the simplest solution to the one dimensional Helmholtz equation introduced by Durnin in 1987, researchers of the photonics community has extensively studied the subject further. Over the years a few techniques have been proposed to generate these beams experimentally. One of the most well known method introduced by Durnin himself, is the annulus method whereby a lens and ring slit aperture is used to produce such beams [1].

Other methods include using a conical lens known as an axicon $[2,3]$. This is the most efficient method since there is almost a hundred percent transmission of the initial beam intensity that produces the Bessel beam. A more advance method is using holography which involves the use of diffractive optical elements. Recently the development of spatial light modulators has lead to generation of not only Bessel beam but numerous other novel beams as well [4].

The interesting characteristic of Bessel beams are that they non-diffracting as well as self-reconstructive within their validity region [3]. They downfall is that they exist for a finite distance hence outside their boundary region a Bessel function undergoes a spatial transformation and forms a conical field.

Here we will demonstrate the generation of zero- and higher-order Bessel beams using a conical lens. We will also outline a method that overcomes the limitation of propagation distance hence instead of obtaining an annular ring in the far-field the Bessel beam will retain its spatial spectrum to form a Bessel beam in the far-field as well [5].

\section{GENERATION OF BESSEL BEAMS USING A SINGLE CONICAL LENS}

Mathematically Bessel beams exists over an infinite area carrying an infinite amount of power. This ideal case however is not reproducible experimentally. As mentioned previously there are a few techniques to demonstrate the existence of Bessel beams however due to its efficiency an axicon is chosen to demonstrate the generation of these beams as illustrated in Fig. 1.

\footnotetext{
† Corresponding author: Andrew Forbes; tel: +27 12841 2368; fax: +27 12841 3152; email: aforbes1@csir.co.za

Laser Beam Shaping XI, edited by Andrew Forbes, Todd E. Lizotte, Proc. of SPIE Vol. 7789, 77890C · @ 2010 SPIE · CCC code: 0277-786X/10/\$18 · doi: 10.1117/12.863345
} 


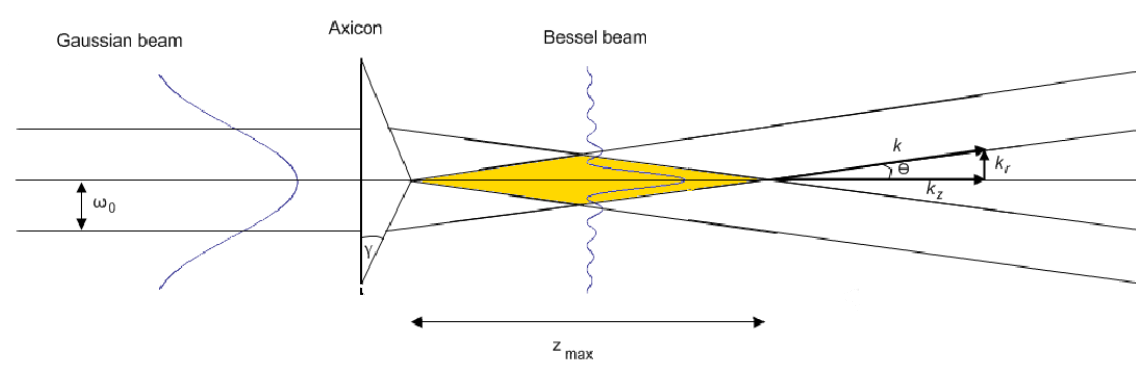

Figure 1: Schematic of the optical design to demonstrate the existence of Bessel beams using an axicon lens.

An axicon lens with a cone angle of $5^{0}$ is illuminated by a Gaussian beam. Since an axicon is a conical lens, the light rays propagate along a cone to form a diamond shaped region in which interference occurs and hence a Bessel beam is produced. Unlike a Gaussian beam which are propagation invariant, Bessel beams exist for a finite region which is inversely proportional to the divergence defined by (1),

$$
z_{\max }=\frac{k}{k_{r}} \omega_{0} \approx \frac{\omega_{0}}{\theta}
$$

where wo is the beam radius and $\theta$ is the divergence defined by Eq.(2)

$$
\theta=(n-1) \gamma
$$

Eq. 2 confirm that Bessel beams are non-diffracting within its validity region however at the boundary they undergo a transformation from a Bessel function (near-field profile) to a conical field characterized as an annular ring intensity distribution (far-field profile). The above optical design was incorporated into an experimental setup whereby the theoretical as well as experimental results are depicted in Fig. 2. As observed there is a good agreement between the theoretical and experimental results obtained.

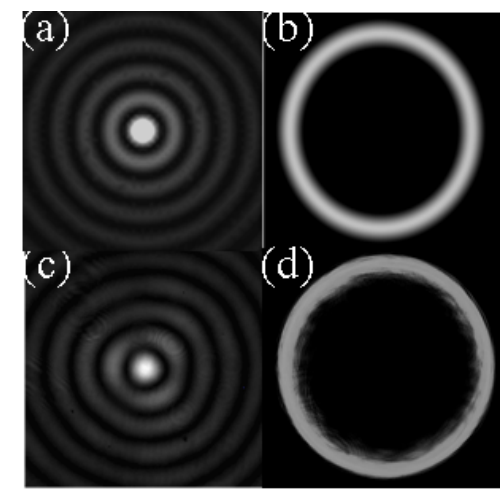

Fig.2: Theoretical as well experimental generation of zero-order Bessel beam: (a) theoretical image of zero-order Bessel beam (near-field profile), (b) theoretical image of the zero-order Bessel beam (far-field profile), (c) experimental image of a zero-order Bessel beam (near-field profile) and (d) experimental image of a zero-order Bessel beam (far-field profile).

Taking this a step further and illuminating the axicon lens with higher-order Laguerre Gaussian (LG) beams instead of a Gaussian beam leads to the generation of higher-order Bessel beams. LG beams carry orbital angular momentum (OAM) which is dependent on the order of the topological charge, $l$. They are generated using a spatial light modulator. The grayscale phase pattern as well as the corresponding LG beam obtained experimentally is illustrated in Fig. 3. 


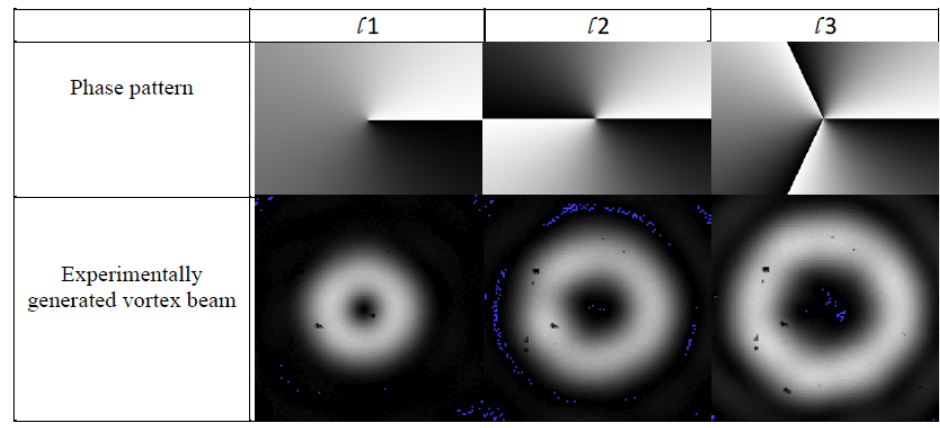

Fig.3: Grayscale phase patterns for the different order vortex beams as well as the corresponding vortex beam.

It can be deduced that Higher-order Bessel beams are composed of a Bessel beam engulfed by a LG Beam hence they also carry OAM. Fig.4 illustrates the experimental results obtained for different higher-order Bessel beams in the nearand far-field.

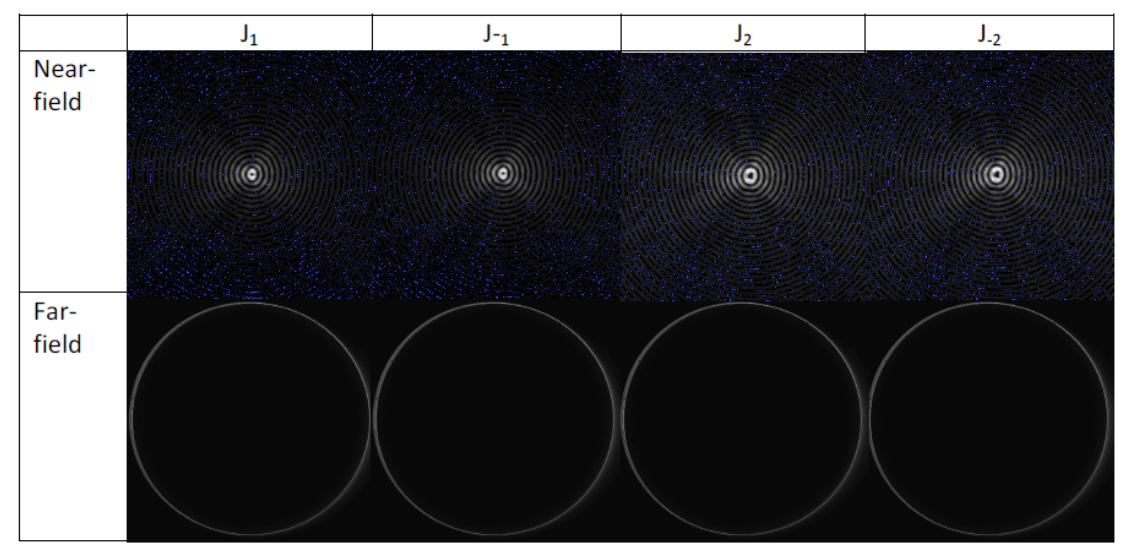

Fig.4: Near- and far-field images of Higher-order Bessel beams.

The higher the order of the Bessel beam, the greater the amount of OAM carried by the beam. As in the case of zeroorder Bessel beams, higher-order Bessel beams also exist for a finite region as well as they are non-diffracting.

\section{Z-DEPENDENT BESSEL-LIKE BEAMS}

This abrupt change in intensity distribution from the near- to far-field can be considered a disadvantage to such beam and can be avoided using a double axicon and lens system illustrated in Fig.5. Upon propagating a well collimated Gaussian beam through axicon, $\mathrm{Ax}_{1}$ and a spherical lens of focal length $\mathrm{F}$, a conical field is produced. The conical field thereafter passes through the second axicon, Ax $x_{2}$ which refracts the light rays in the direction of beam propagation such that the crossing angles, $\theta$,decreases as the propagation distance increases. This implies that a Bessel function is still produced as $\mathrm{z} \rightarrow \infty$. 


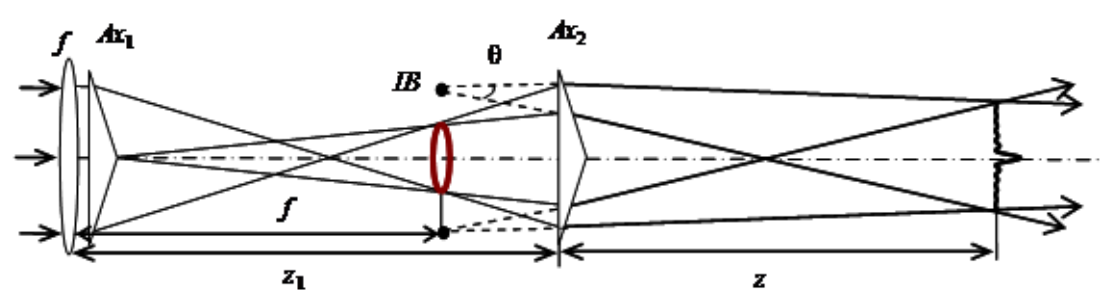

Fig.5: Optical design to generate z-dependent Bessel-like beams

\section{CONSIDERING A MATHEMATICAL APPROACH TO EXPLAINING Z-DEPENDENT BLB'S}

The stationary phase method is implemented in order to confirm that the field entering the second axicon is conical. From Fresnel integral the field at the input plane of the second axicon is defined by Eq. 3,

$$
a_{1}\left(\rho, z_{1}\right)=-\frac{i}{\lambda z_{1}} \exp \left(\frac{i k_{0} \rho^{2}}{2 z_{1}}\right)_{0}^{2 \pi} \int_{0}^{2 \pi R_{a 2}} \exp \left(-\frac{\rho_{1}^{2}}{\rho_{0}^{2}}-i k_{0} \gamma_{2} \rho_{1}-\frac{i k_{0} \rho \rho_{1}}{z_{1}} \cos \left(\varphi-\varphi_{1}\right)\right) \rho_{1}^{2} d \rho_{1} d \varphi_{1} \quad,
$$

Where $\mathrm{w}_{0}$ is the beam radius of the Gaussian beam, $\mathrm{k}_{0}$ is the wave number is given by Eq. 4

$\frac{1}{\rho_{0}^{2}}=\frac{1}{w_{0}^{2}}+\frac{i k_{0}}{2 F}-\frac{i k_{0}}{2 z_{1}}$,

Thereafter applying the stationary phase method, the above equation yields:

$a_{1}\left(\rho, z_{1}\right)=-\frac{i \rho F^{2}}{\left(z_{1}-F\right)^{2}}\left(1-\frac{\gamma_{2} z_{1}}{\rho}\right)^{3 / 2} \exp \left[\frac{i k_{0}}{2 z_{1}}\left(\rho^{2}+\frac{z_{1} / F-1+i z_{1} / z_{0}}{\left(z_{1} / F-1\right)^{2}+\left(z_{1} / z_{0}\right)^{2}}\left(\rho-\gamma_{2} z_{1}\right)^{2}\right)\right]$.

As calculated above the field some arbitrary distance $\mathrm{z}$ after the second axicon is thereafter determined from Eq. 6

$$
\begin{aligned}
a_{2}(\rho, z)= & -\frac{i}{\lambda z} \int_{0}^{2 \pi R_{a 2}} \int_{0} a_{1} \exp \left(\frac{i k_{0}\left(\rho^{2}+\rho_{1}^{2}-2 \rho \rho_{1} \cos \left(\varphi-\varphi_{1}\right)\right)}{2 z}-i k_{0} \gamma_{2} \rho_{1}\right) \rho_{1}^{2} d \rho_{1} d \varphi_{1} \\
& =\exp \left(\frac{i k_{0} \rho^{2}}{2 R(Z)}\right)\left[g_{+}(\rho, z) \exp \left(i k_{0} \gamma(z) \rho\right)-i g_{-}(\rho, z) \exp \left(i k_{0} \gamma(z) \rho\right)\right] .
\end{aligned}
$$

Where

$$
\begin{aligned}
& g_{ \pm}(\rho, z)=\frac{f}{2\left(z+z_{1}-f\right)} \sqrt{\gamma_{2}-\gamma_{1}\left(1+\frac{z_{1}}{z}\right) \pm 1}, \\
& R(z)=z\left[1+\frac{z}{z_{1}-f}\right],
\end{aligned}
$$


and we define:

$$
\gamma(z)=\frac{\gamma_{2} z_{1}+\left(\gamma_{1}-\gamma_{2}\right) f}{z_{1}+z-f}
$$

From the zero-order Bessel function of the first kind, $J_{0} \approx \sqrt{\frac{2}{\pi z}} \cos \left(z-\frac{\pi}{4}\right)$, Eq. 10 is attained

$$
a_{2}(\rho, z) \approx \frac{1}{2} \sqrt{\frac{\pi k_{0} \gamma(z) \rho}{2}}\left(g_{+}(\rho, z)+g_{-}(\rho, z)\right) \exp \left[\frac{i k_{0}}{2}\left[\frac{\rho^{2}}{z}+\frac{\rho^{2}}{R(z)}-\gamma^{2}(z) R(z)\right] J_{0}\left[k_{0} \gamma(z) \rho\right]\right. \text {. }
$$

Eq. 9 clearly shows that the cone angle, $\gamma$, is dependent upon the longitudinal distance $\mathrm{z}$ as well as it confirms that an increase in $\mathrm{z}$ will lead to decrease in the cone angle. Similarly the above mathematical approach can be carried out on higher-order z-dependent BLB's [6].

\section{EXPERIMENTALLY GENERATED Z-DEPENDENT BESSEL-LIKE BEAMS}

From the above in can be deduced that these beams are Bessel-like hence they are so named z-dependent Bessel-like beams. These beams are produced however at the expense of losing its non-diffracting nature which will be validated shortly. Incorporating the optical design outlined in Fig. 5 into an experimental setup results to the generation of BLB's in the near- and far-field illustrated in Fig. 6.

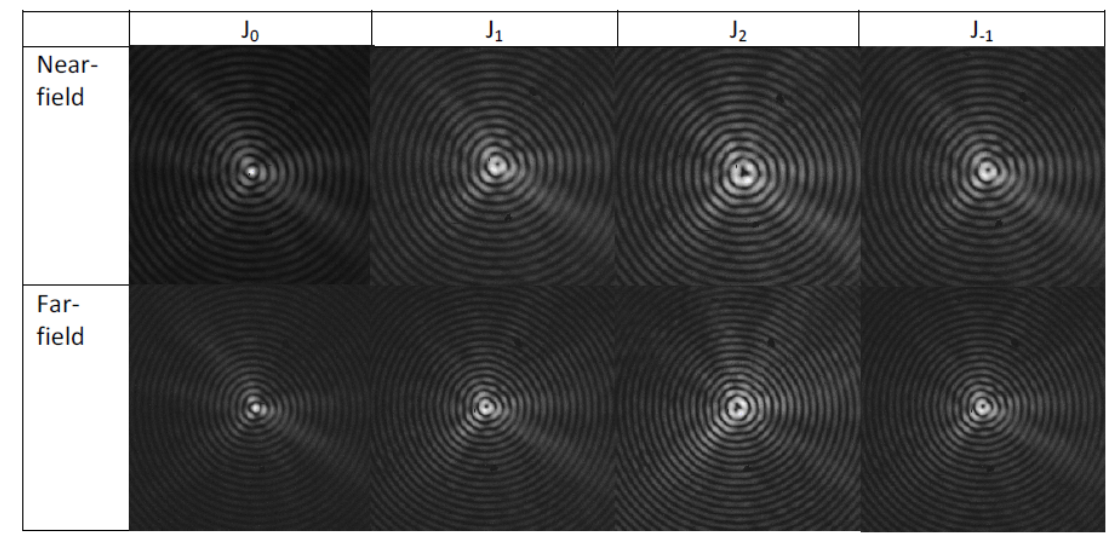

Fig. 6: z-dependent Bessel-like beams.

As mentioned previously z-dependent BLB's are produced at the loss of their non-diffracting nature. An experimental system was design to validate the above statement. It was observed that there is a linear increase in diffraction as the propagation distance was increased. The results obtained are illustrated in Fig. 7. It can be deduced that the higher the order of the BLB'S the quicker the beam diffracts. 


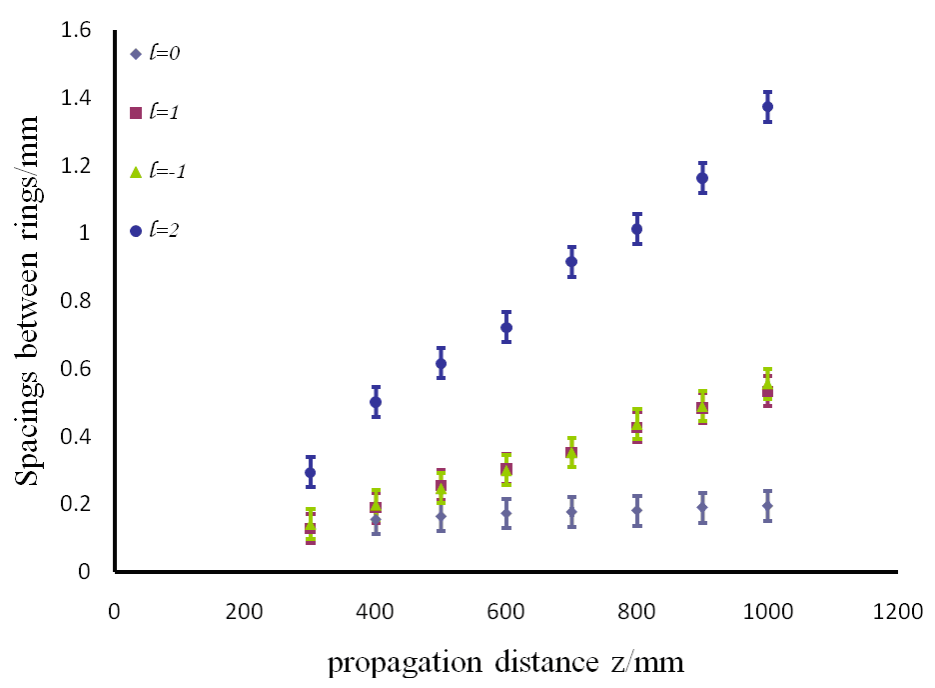

Fig. 7: Graph illustrating the divergence of z-dependent Bessel-like Beams.

\section{CONCLUSION}

Here it was shown that by the use of ordinary optical elements found in the laboratory, a new method for generating Bessel beams in the far-field is possible. This was achieved by basically varying the cone angle such that as $\mathrm{z} \rightarrow \infty, \gamma \rightarrow 0$ hence zero- and higher-order Bessel-like beams in the far-field are easily reproducible. Conclusively this new design for generating long range z-dependent Bessel-like beams can be an advantage for many applications especially in optical trapping.

\section{REFERENCES}

[1] Durnin J., "Exact solution for nondiffracting beams: I. The scalar theory," J.Opt. Soc. Am, B 4(4), 651-654 (1987).

[2] Litvin I.A., McLaren M.G., and Forbes A., "A conical wave approach to calculating Bessel-Gauss beam reconstruction after complex obstacles," Opt. Commun., 282, 1078-1082 (2009).

[3] Bouchal z., Wagner J. and Chulup M.,"Self-reconstruction of a distorted nondiffracting beam," Opt. Commun. 151(4-6), 207-211 (1998)

[4] Vasara A., Turunen J. and Friberg A.T., "Realization of general nondiffracting beams with computer-generated holograms," J. Opt. Soc. Am. A6(11), 1748-1754 (1989)

[5] Belyi V., Forbes A., Kazak N., Khilo N. and Ropot P.,"Bessel-like beams with z-dependent cone angles," Opt. Express 18,1966-1973 (2010)

[6] Belyi V., Forbes A., Kazak N., Khilo N. and Ropot P.,"Higher-order z-dependent-Bessel-like beams,” In preparation 